\title{
Incidence of Multidrug Resistant Pseudomonas Aeruginosa Isolated From Burn Patients Tertiary Care Hospital, Jamnagar, Gujarat, India.
}

\author{
Pooja A. Kamaria*, Binita J. Aring**, Mala Sinha*** \\ *Resident, **Associate Professor, *** Professor \& Head. Microbiology department, Shri M.P. Shah \\ Government Medical College and GGG Hospital, Jamnagar, Gujarat.
}

\begin{abstract}
:
Background: Pseudomonas aeruginosa is a ubiquitous micro-organism that can rapidly acquire resistance to different broad-spectrum antibiotics. Multidrug resistant (MDR) Pseudomonas aeruginosa is an emerging cause of mortality and morbidity in burn patients, which causes 4-60\% nosocomial infections in different parts of the world. ${ }^{1}$

Objectives:

- To isolate Pseudomonas aeruginosa from pus samples of burns patients.

- To assess the rates of antibiotic resistance and multidrug resistance among Pseudomonas isolates.

Material \& Methods: This study was carried out in the Department of Microbiology, Shri M. P. Shah Government Medical College, Jamnagar, Gujarat from September 2014 to June 2015 from burns ward, Guru Govind singh Govt. Hospital Jamnagar. A total of 391 swab samples were tested. The Pseudomonas aeruginosa isolates were identified by their direct microscopy examination, colony morphologies, pigment, Gram's staining patterns, oxidase test, catalase test, Oxidation fermentation test, Triple sugar iron agar test, Arginine dihydrolase and Sugar fermentation test. Antibiotic sensitivity was determined for Amikacin (30mcg), Netilmycin(30mcg),Gentamycin(10mcg),Ceftazidime(30mcg),Piperacillin(100mcg), $\quad$ Piperacillin + Tazobactum(100mcg/10mcg), Aztreonem (30mcg), Imipenem (10mcg), Meropenem (10mcg), Colistin (10mcg), Polymixin B(300units), Ciprofloxacin(5mcg).

Results: Incidence of Pseudomonas aeruginosa from burns patients is 36.45\%. Incidence of Multidrug Resistant (MDR) Pseudomonas aeruginosa is 36.48\%. Antibiotic susceptibility tests showed high level resistance toGentamycin(93.24\%), Ciprofloxacin(89.18\%), Amikacin(85.13\%), Aztreonem(83.78\%), Ceftazidime(81.08\%), Netilmicin(75.67\%), Piperacillin(72.97\%), Piperacillin+Tazobactum(56.76\%), Imipenem(14.86\%). Resistance to Colistin and Polymixin B were 0\%.

Conclusions: We conclude that Pseudomonas aeruginosa with high level resistance to Aminoglycosides, Fluoroquinolones \& Penicillin group of drugs. Infection in burns continues to be a great problem which is not yet solved and posses a challenge to the microbiologist and the surgeon. Based on the result of the antibiotic susceptibility testing of the various isolates antibiotics are to be administered only on clinical suspicion of sepsis.
\end{abstract}

Keywords: Burn, Swab samples, Pseudomonas aeruginosa, Multidrug Resistance,

\section{Introduction}

Pseudomonas is a non-fermentative gram negative bacterium, which is widely distributed in nature. It is responsible for about $10 \%-20 \%$ of nosocomial infections which are seen as septicemia in burn and wound infections, cystic fibrosis intensive-care units (ICUs), etc [1].

Pseudomonas is a ubiquitous micro-organism that can rapidly acquire resistance to different broadspectrum antibiotics. Multidrug resistant (MDR) Pseudomonas is an emerging cause of mortality and morbidity in burn patients, which causes 4-60\% nosocomial infections in different parts of the world [1].

The morbidity and mortality associated with Pseudomonas are mainly attributed to inadequate empirical therapy and/ or delay in the initiation of appropriate therapy [2,3]. Burns Patients with Pseudomonas infection have increased need for debridement and they frequently require re-grafting due to loss of skin grafts or allografts [4].

MDR Pseudomonas elaborates inactivating enzymes that make beta-lactams and carbapenems ineffective, such as extended spectrum beta lactamases (ESBLs) and metallo- $\beta$-lactamases (MBLs) [5]. MDR Pseudomonas is defined as a bacterium which is resistant to anti-microbial agents which are included in three or more anti-Pseudomonal anti-microbial classes (carbapenems, fluoroquinolones, penicillins /cephalosporins and aminoglycosides) [6]. Pseudomonas spp. Continues to be a leading cause of nosocomial infection which is often 
life threatening. Natural resistances to this make the treatment problematic. Indiscriminate use of antibiotics has led to the emergence of multiple drug resistance strains [7].

\section{Material And Methods}

Study duration \& sample size: This study was carried out in the Department of Microbiology, Shree M.P. Shah Govt. Medical College and G.G.G. Hospital, Jamnagar, Gujarat from September 2014 to June 2015. The study group comprised 391 swab samples of 203 patients, who were admitted in burns ward in Guru Gobind Singh Govt. Hospital, Jamnagar.

Collection of Samples: Pus samples from burns patients received in Microbiology Department, Shree M.P. Shah Medical College and G.G. Hospital, Jamnagar were tested to isolate Pseudomonas aeruginosa to detect antimicrobial resistance pattern. The pus samples were collected onto sterile cotton wool swabs aseptically. All samples were transferred to laboratory soon after being obtained. Total 391 swab samples were tested during study period. The samples were cultured and smear made for direct microscopy.

\section{$1^{\text {st }}$ Day Follow Up:}

1) Direct Microscopy:

Smears were made on glass slide from pus samples for gram staining technique. Smears were allowed to air dry, heat fixed and stained by gram staining technique. The stained slides were examined microscopically under oil immersion lens for pus cells and bacteria.

\section{Result of Direct Microscopy:}

From all the smears gram positive and gram negative results were observed. Pseudomonas aeruginosa appear pink -red bacillus, $1.5-3 \mu \mathrm{m} \times 0.5 \mu \mathrm{m}$.

\section{2) Culture:}

All the samples, either gram negative bacilli or gram positive cocci in smear examination were inoculated on MacConkey agar and Blood agar plates. The culture plates were incubated aerobically for $24 \mathrm{hrs}$ at $37^{\circ} \mathrm{c}$. Growth and cultural characteristics were observed next day.

\section{$2^{\text {nd }}$ Day Follow Up: \\ Colony characteristics:}

It grows well on ordinary media, producing large, opaque, irregular colonies with a distinctive, musty or earthy smell. On MacConkey media, it produced non-lactose fermenting, Pale colonies. Many strains are hemolytic on blood agar. They also produce pigments best known is pyocyanin. From colony, gram staining and motility by hanging drop preparation were also done. On hanging drop preparation method Pseudomonas aeruginosa is actively motile by a polar flagellum.

\section{Biochemical Reaction:}

Oxidase test was positive, Catalase test was positive, Arginine dihydrolase was positive. Oxidation fermentation test shows that test was oxidative for Pseudomonas aeruginosa. In triple sugar iron agar test, it was alkaline slant/ alkaline butt(K/K reaction). In Sugar fermentation test - Glucose is utilized oxidatively, forming acid only. Indole, MR, VP and $\mathrm{H} 2 \mathrm{~S}$ test are negative.

\section{Antibiotic Sensitivity Testing:}

Anti-microbial sensitivity testing was done according to the CLSI (Clinical and Laboratory Standards Institute) guidelines for Amikacin(30 mcg), Netilmycin(30mcg), Gentamycin(10mcg), Ceftazidime(30mcg), Piperacillin(100mcg), Piperacillin+Tazobactum(100mcg/10mcg), Aztreonem(30mcg), Imipenem(10mcg), Meropenem(10mcg), Colistin(10mcg), Polymixin B(300units), Ciprofloxacin(5mcg).

Ethical clearance: it is a retrospective analysis of samples tested for routine laboratory diagnosis; hence ethical clearance is not necessary.

\section{Results}

Sex wise distribution of patients suggests that out of 203 patients $78(38.42 \%)$ were male and $\mathbf{1 2 5}(\mathbf{6 1 . 5 8 \%})$ were female. Age wise distribution of patients suggests that highest cases were found in age group of $\quad 21-\mathbf{4 0}(\mathbf{5 3 . 2 0} \%)$ followed by age group $41-60(24.63 \%)$. Table-1 shows age group infected with Pseudomonas aeruginosa, highest in the age group $21-\mathbf{4 0}(\mathbf{5 2 . 7 0 \%})$ followed by $41-60(28.38 \%)$. Table-2 shows that incidence rate of Pseudomonas aeruginosa \& incidence rate of MDR Pseudomonas aeruginosa, isolated from burns patients which are $\mathbf{3 6 . 4 5 \%} \& \mathbf{3 6 . 4 8 \%}$ respectively. Table-3 shows antibiotic resistant 
pattern of Pseudomonas aeruginosa. It showed high level resistance to Gentamycin $(93.24 \%)$, Ciprofloxacin(89.18\%), Amikacin(85.13\%), Aztreonem(83.78\%), Ceftazidime(81.08\%), Netilmicin(75.67\%), Piperacillin(72.97\%), Piperacillin+Tazobactum(56.76\%), Imipenem(14.86\%). Resistance to Colistin and Polymixin B were $0 \%$.

\section{Discussion}

There has been rapid emergence of MDR P.aeruginosa in recent times, which is an important concern for clinicians who treat these infections. In the present study, incidence rate of Pseudomonas aeruginosa is $\mathbf{3 6 . 4 5 \%}$. A study carried out by Navendu et al. in 2006 showed that $\mathbf{3 5 . 7 5 \%}$ of Pseudomonas aeruginosa among infected burns patients, which is almost similar to present study [8]. A study carried out by Heggers et al. in USA in 1998 showed that $\mathbf{5 1 \%}$ of the burns patients are having infection of Pseudomonas aeruginosa, which has higher rate of infection as compare to present study [9].

Pseudomonas aeruginosa shows highest resistance to gentamicin(93.24\%). A study done by Navendu et al., Saha et al., Indu at al., showed $81.6 \%, 80.77 \%$ \& $81.03 \%$ resistance to gentamicin respectively[8,11,12]. Present study shows that resistance to ceftazidime is $81.08 \%$, similar study done by Indu et al.obtained $70.68 \%$ of resistance [12].According to Srinivasan et al. P. aeruginosa was 100\% resistant to ceftazidime[10].

Present study shows that resistance to ciprofloxacin is $89.18 \%$, similar study done by Ashwin et al. \& Saha et al. showed $90.62 \%$ \& $92.16 \%$ of resistance respectively[13,11]. Present study shows that resistance to imepenem is $14.86 \%$, similar study done Indu et al., obtained $18.9 \%$ of resistance to imepenem[12]. Present study shows that resistance to colistin is $0 \%$, similar study done by Indu et al. obtained $0 \%$ of resistance [12].

In the present study, MDR Pseudomonas rate (resistance to anti-microbial agents which are included in three or more anti-Pseudomonal anti-microbial classes (carbapenems, fluoroquinolones, penicillins /cephalosporins and aminoglycosides [6]) is $\mathbf{3 6 . 4 8 \%}$. A study done by Unan et al.,[14] in Turkey reported rates of MDR, which were as high as $\mathbf{6 0 \%}$, whereas study done by Sabir et al., in Pakistan detected lower rates of MDR that was $\mathbf{2 2 . 0 8 \% ~ [ 1 5 ] . ~ H o w e v e r , ~ r a t e s ~ o f ~ o u r ~ s t u d y ~ a r e ~ c o m p a r a b l e ~ t o ~ a ~ s t u d y ~ d o n e ~ i n ~ E g y p t , ~ w h e r e ~ G a d ~}$ et al., observed $\mathbf{3 6 \%}$ MDR P. aeruginosa [16]. It is also comparable to a study done by Indu et al. that was $36.2 \%$ [12].

Pseudomonas aeruginosa has gradually become a major cause of nosocomial infections which occur in burn patients and which requires immediate and effective implementation of infection control strategies, to combat its spread. Environmental sources may play a significant role in spread of MDR among hospitalized patients. In current times, antibiotics with anti Pseudomonal activity which are available include the aminoglycosides, ticarcillin, ureidopenicillins and ciprofloxacin. Combination treatments are generally recommended for suspected Pseudomonas infections. It has been reported that the choice of cabapenem, cefepime or piperacillin + tazobactum, ciprofloxacin and gentamicin combinations with amikacin or tobramycin, in current times, appears to provide the widest potential antimicrobial activity against MDR P. aeruginosa [16].

The lack of any new compounds in the near future indicates that national and local surveillance efforts are essential, to provide clinicians with correct information for choosing right antimicrobial therapy. Rigorous monitoring for MDR among Pseudomonas isolates is very important, because outbreaks caused by strains which are resistant to potentially useful agents, including carbapenems, have been reported elsewhere [17-19].

\section{Conclusion}

We conclude that Pseudomonas aeruginosa with high level resistance to Aminoglycosides, Fluoroquinolones \& Penicillin group of drugs. Infection in burns continues to be a great problem which is not yet solved and poses a challenge to the microbiologist and the surgeon. Restriction of 'selected antibiotic usage' and/or infection control policies must be tailored for each institution, to combat the rapid emergence of MDR P. aeruginosa in burn patients. The lack of newer antimicrobial agents with activities against P. aeruginosa, makes periodic studies on the antimicrobial esistance patterns very important.

\section{References}

[1]. Carmeli YN, Troillet G, Eliopoulos GM, Samore MH. Emergence of antibiotic resistant Pseudomonas aeruginosa: Comparison of risks associated with different Anti pseudomonal agents. Antimicrob. Agents Chemother. 1999; 3:1379-82 Lewis GJ, Fang X, Gooch M, Cook PP. Decreased resistance of Pseudomonas aeruginosa with restriction of ciprofloxacin in a large teaching hospital's intensive care and intermediate care units. Infect Control Hosp Epidemiol. 2012;33:368-73.

[2]. Obritsch MD, Fish DN, MacLaren R, Jung R. National surveillance of antimicrobial resistance in Pseudomonas aeruginosa isolates obtained from intensive care unit patients from 1993 to 2002. Antimicrob Agents Chemother. 2004;48:4606-10.

[3]. Coetzee E, Rode H, Kahn D. Pseudomonas aeruginosa burn wound infection in a dedicated paediatric burns unit. S Afr J Surg. 2013;51:50-53.

[4]. Vahdani M, Azimi L, Asghari B, Bazmi F, Rastegar LA. Phenotypic screening of extended-spectrum ß-lactamase and metallo-ßlactamase in multidrug-resistant Pseudomonas aeruginosa from infected burns. Ann Burns Fire Disasters.2012;25(2):78-81.

[5]. Magiorakos AP. Multidrug Resistant (MDR), Extensively Drug Resistant (XDR) and Pandrug-1 Resistant (PDR) bacteria in healthcare settings. Expert Proposal for Standardized International Terminology, 2011. Available online at www.escmid org.

[6]. Dr. Bibhabati Mishra: Resistance pattern of Pseudomonas species, 2001, PP.3 
Incidence Of Multidrug Resistant Pseudomonas Aeruginosa Isolated From Burn Patients Tertiary..

[7]. Navendu M.J. : Prevalence of bacterial infection in patients of burns with their antibiotic sensitivity pattern -2006 .

[8]. Heggers JP., Good Heart R., - Is the limulus amebocyte lysate the sole predictor of septic episodes in major thermal injuries, J. Burn, Care Rehabil 1998, Nov.-Dec.; 19(6):512-5.

[9]. Srinivasan S, Vartak AM, Patil A, Saldanha J. Bacteriology of burn wound at the Baba Jerbai Wadia hospital for children, MumbaiA 13 year study. Indian J Plastic Surg. 2009;42(2):213-8.

[10]. Saha SK, Mauzzam N, begum SA, Chowdhury A, Islam MS, Parveen R. Study on time related changes in aerobic bacterial pattern of burn wound infection. Faridpur Med. Coll J 2011;6(1):41-5.

[11]. Indu B. - Incidence of multidrug resistant pseudomonas aeruginosa isolated from burn patients and environment of teaching institute. 2014.PMCID: PMC4080000.

[12]. Ashwin N.A. 'Detection of Extended spectrum Beta lactamase produces among surgical wound infections and burn patients in Jipmer. I.J. of M.M.(2000), 18(4):160-165.

[13]. Unan D, Gnseren F. The resistance of P. aeruginosa strains isolated from nosocomial infections against various antibiotics. Mikrobiyol Bult.2000;34:255-60.

[14]. Sabir R, Alvi SFD,FAwwad A. Antimicrobial susceptibility pattern of aerobic microbial isolates in a clinical laboratory in KarachiPakistan. Pak J Med Sci. 2013;29(3):851-5.

[15]. Gad GF, EI-Domany RA, Zaki S, Ashour HM. Characterization of Pseudomonas aeruginosa isolated from clinical and environmental samples in Minia, Egypt: Prevalance, Antibiogram and Resistance mechanisms. J Antimicr chemother. 2007;60:1010-7

[16]. Ramprasad BP, Marissa R, Suprama D. Role of Pseudomonas in Nosocomial infections and Biological Characterization of Local Strains. J Biosci Tech. 2010;1(4):170-9.

[17]. Pfaller MA, Jones RN, A review of the in vitro activity of meropenem and comparative antimicrobial agents tested against 30,254 aerobic and anaerobic pathogens isolated worldwide. Diagn Microbial Infect Dis. 1997;28:157-63.

[18]. Pfaller MA, Jones RN, Biedenbach DJ, the MYSTIC Program Study Group(USA). Antimicrobial resistance trends in medical centers using carbapenems: report of 1999 and 2000 results from the MYSTIC program (USA). Diagn Microbial Infect Dis. 2001;41:177-82.

Table - 1 Age Group Infected With Pseudomonas Aeruginosa Strains

\begin{tabular}{|l|l|l|}
\hline Age group (Years) & No. of Pseudomonas aeruginosa strains & Percentage (\%) \\
\hline $0-20$ & 9 & $12.16 \%$ \\
\hline $21-40$ & 39 & $52.70 \%$ \\
\hline $41-60$ & 21 & $28.38 \%$ \\
\hline$>60$ & 5 & $6.76 \%$ \\
\hline
\end{tabular}

Table - 2 Incidence Rate of Pseudomonas Aeruginosa \& Incidence Rate Of Mdr Pseudomonas Aeruginosa Isolated From Burns Patients

\begin{tabular}{|l|l|l|l|}
\hline Total patients & Pseudomonas aeruginosa isolated & Incidence rate & MDR Incidence rate \\
\hline 203 & 74 & $36.45 \%$ & $36.48 \%$ \\
\hline
\end{tabular}

Table - 3 Antibiotic Resistant Pattern of Pseudomonas Aeruginosa

\begin{tabular}{|l|l|}
\hline Name of antibiotics & Resistance (\%) \\
\hline Piperacillin (PI) & 72.97 \\
\hline Ceftazidime (CAZ) & 81.08 \\
\hline Gentamicin (GEN) & 93.24 \\
\hline Piperacillin+Tazobactum(PT) & 56.76 \\
\hline Aztreonem (AT) & 83.78 \\
\hline Imipenem (IMP) & 14.86 \\
\hline Amikacin (AK) & 85.13 \\
\hline Ciprofloxacin (CIP) & 89.18 \\
\hline Netilmicin (NET) & 75.67 \\
\hline Polymixin B (PB) & 0 \\
\hline Colistin (CL) & 0 \\
\hline
\end{tabular}

